\title{
Feature Level Fusion of Palm and Face for Secure Recognition
}

\author{
Gayatri Umakant Bokade and Ashok. M. Sapkal
}

\begin{abstract}
Biometric user authentication techniques for security and access control have evoked an enormous interest by science, industry and society in the last two decades. But even the best single biometric system suffers from spoof attacks, intra-class variability, noise, susceptibility etc. In the realm of biometrics, the consolidation of evidence presented by multiple biometric sources is an effective way of enhancing the recognition accuracy of an authentication system. This paper proposes an authentication for a multimodal biometric system identification using two traits i.e., face and palmprint at feature extraction level. The training database consists of face and palmprint images. Principal Component Analysis method is used to extract the features from face and palmprints separately. The feature normalization and feature concatenation scheme followed by a dimensionality reduction procedure is adopted to form the feature matrix. The normalized match (distance) scores generated by respective palm and face features before fusion are used to form fused match score. The Euclidean distance and the feature distance are calculated after fusion. All three distances are used to arrive at final decision. Feedback routine implemented between the feature extraction and the matching modules of the biometric system can lead to substantial improvement in multimodal matching performance.
\end{abstract}

Index Terms-Biometrics, face, fusion, match score, palmprint.

\section{INTRODUCTION}

In multibiometric fusion [1], feature level fusion [2], [3] makes use of integrated feature sets obtained from multiple biometric traits. Fusion at feature level [2], [3] is found to be useful than other levels of fusion such as match score fusion [4], decision fusion [4], rank level fusion [4]. Since feature set contains relevant and richer information about the captured biometric evidence, fusion at feature level is expected to provide more accurate authentication results. It is very hard to fuse multiple biometric evidences [2], [3] at feature extraction level in practice because the feature sets are sometimes found to be incompatible. Apart from this reason, there are two more reasons to achieve fusion at feature extraction level such as the feature spaces are unknown for different biometric evidences and fusion of feature spaces may lead to the problem of curse of dimensionality [2].

Manuscript received February 15, 2012; revised March 15, 2012. This work was done as one of the method of fusion during completion of studies of Master of Technology, at College of Engineering Pune.

The authors are with College of Engineering Pune, an Autonomous Institute of Government of Maharashtra. She is now with Nutan Maharashtra Institute of Engineering and Technology, Talegaon Dabhade, University of Pune, Maharashtra, India(e-mail: gsambadkar@gmail.com; ams.extc@ coep.ac.in).
Further, poor feature representation may cause to degrade the performance of recognition of users.

Multimodal systems [4] acquire information from more than one source. Unibiometric identifiers [5] use single source biometric evidence and often are affected by problems like lack of invariant representation, non-universality, noisy sensor data and lack of individuality of the biometric trait and susceptibility to circumvention. These problems can be minimized by using multibiometric systems.

Consolidate evidences obtained from multiple biometric sources. Feature level fusion [2] of biometric traits is a challenging problem in multimodal fusion. However, good feature representation and efficient solution to curse of dimensionality problem can lead to feature level fusion with ease. Multibiometrics fusion [4] at match score level, decision level and rank level have extensively been studied and there exist a few feature level fusion approaches. However, to the best of the knowledge of authors, there is enough scope to design an efficient feature level fusion approach.

The paper is organized in the following way.

Section 2 gives system architecture. Section 3 gives feature extraction from palm and face images. Section 4 gives feature fusion of both the traits and section 5 identification process.

\section{SYSTEM ARCHITECTURE}

The system architecture consists of five major blocks, image acquisition module, image pre-processing module, feature extraction and fusion module and the identification module.

Image acquisition module is designed in the palmprint laboratory. It provides the same background and intensity invariant effect while capturing the palm images. Users are asked to put the right hand inside without touching the fingers to each other.

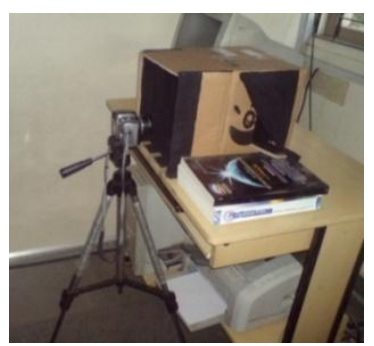

Fig. 1. Image acquisition module.

Image pre-processing module processes the palm images. It performs color to gray transformation, noise reduction, gray to binary transformation and ROI extraction. 


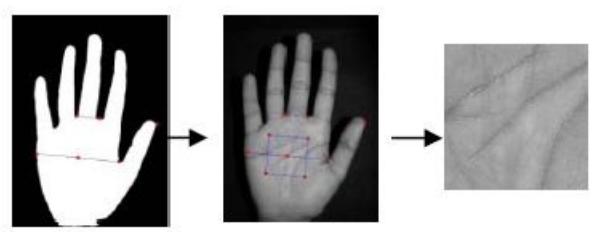

Fig. 2. ROI extraction

Feature extraction and feature fusion module block diagram is shown below:

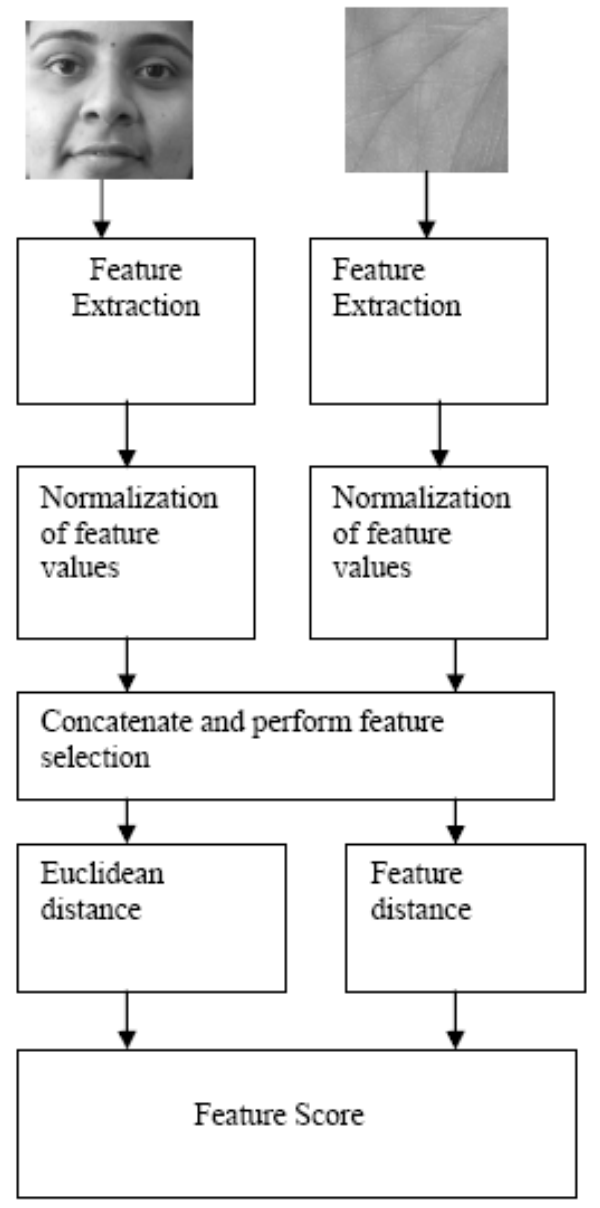

Fig. 3. Block diagram for fusion

The specifications of the digital camera used are:

Canon- Power Shot SX 120 IS

Canon zoom lens $10 \times$ IS

$$
\begin{aligned}
& \text { 6.0-60.0 mm 1:2.8-4.3 } \\
& \text { 10.0 Mega Pixels }
\end{aligned}
$$

Operating Mode: Micro mode, 2.0 Mega pixels.

\section{FEATURE EXTRACTION}

\section{A. Eigenface}

This method uses Principal Components Analysis (PCA) [6] to create a space where faces can be compared against each other for matching. Landmark points are determined on the feature. These points are structural components of the feature, such as the centers of the eyes, so they can be determined on all subjects. The landmark points are then used to normalize the feature in position and size, enforcing a concept of a standard pose. Once the feature is normalized, a mask is then applied around the feature, labeling which pixels are parts of the feature and which are not, determining the data to use for PCA. Ideally, the mask will leave only the sought biometric feature and remove everything else from the image. The shape and size can directly affect the quality of the recognition. A mask that is too small can crop out important structures of the biometric feature. One that is too large can allow meaningless background features (e.g. hair) to be included in the analysis. This can also be achieved by providing the similar background for capturing the face and palm images and then cropping the image to particular size.

After the images in the training set have been normalized and masked, Principal Components Analysis determines eigenvectors F1, F2 ...Fm. The number of eigenvectors $m$ has a maximum value of $\min (\mathrm{R}, \mathrm{C})$, where $\mathrm{R}$ is the number of training images and $\mathrm{C}$ the number of pixels in a training image. The space is tuned to reduce the number of vectors used. Ideally, all possible combinations of the eigenvectors would be explored, using the set which yielded the maximum recognition rate for some validation case. However, this would require the test to be run $2 \mathrm{~m}$ times. Dimension reduction is traditionally performed by dropping vectors from the front (the eigenvector representing the highest variance in the data, usually associated with intensity changes in the images) and from the back (in the direction of the least variance, considered to be negligible).

After PCA is performed, the vectors define a "face space" into which a face I can be projected as a point $\mathrm{I}^{\prime}$ in this space. The image $\mathrm{I}^{\prime}$ is defined as $\mathrm{I}^{\prime}=\alpha 1 \mathrm{~F} 1+\alpha 2 \mathrm{~F} 2+\ldots+\alpha \mathrm{mFm}$. The vector $\alpha=\{\alpha 1 \ldots \alpha m\}$ is the location of the face in the eigenface space.

The following figures shows (a) colored face image, (b) gray face image and (c) Eigen face image

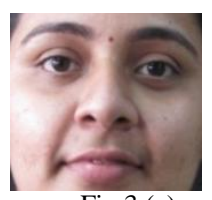

Fig.3 (a)

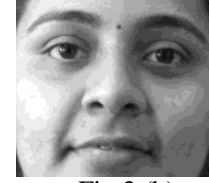

Fig.3 (b)

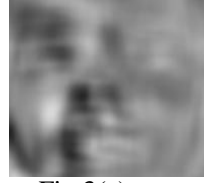

Fig.3(c)

\section{B. Eigenpalm}

The ROI of the palm is rotation invariant and includes features such as the most important principle line features. The palm image is then projected into eigenspace. The projected palm image in eigenspace is represented as image I $=\beta_{1} P_{1}+\beta_{2} P_{2}+\ldots+\beta_{n} P_{n}$. The vector $\beta=\left\{\beta_{1}, \beta_{2} \ldots \beta_{n}\right\}$ is the location of the palm in eigenpalm space. The following figures show a) colored palm image, b) the gray palm image c) Extracted ROI and d) Eigen palm image.

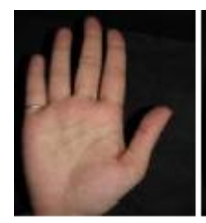

Fig.4 (a)

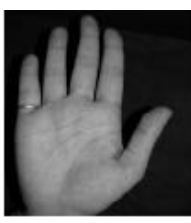

Fig.4 (b)

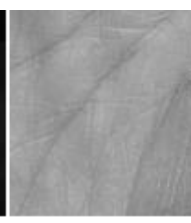

Fig.4(c)

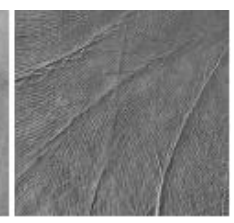

Fig.4 (d)
Principal Component Analysis: PCA has been widely used for dimensionality reduction in computer vision. For the discussion we will consider common notation, image, which 
is applicable to both face and palm images. More formally, let us consider a set of $\mathrm{M}$ images, $\mathrm{i}_{1}, \mathrm{i}_{2} \ldots \mathrm{i}_{\mathrm{M}}$. The average image of the set is defined as $\tilde{\imath}=\frac{1}{M} \sum_{j=1}^{M} i_{j}$. Each image differs from the average image $\hat{\imath}$ by the vector $\varphi_{n}=i_{n}-\hat{\imath}$. A covariance matrix is constructed where: $C=$ $\sum_{j=1}^{M} \varphi_{j} \varphi_{j}^{T}$.Then, eigenvectors, $\mathrm{v}_{\mathrm{k}}$ and eigenvalues, $\lambda_{\mathrm{k}}$ with symmetric matrix $C$ are calculated. The eigenvalue $v_{k}$ determine the linear combination of $\mathbf{M}$ difference images with $\varphi$ to form the eigenimage: $b_{l=\sum_{k=1}^{m} v_{l k}} \varphi_{k}$. where $1=1 \ldots$ M. These Eigen values can be thought as a set of features which together characterized between images from these eigenimages, $\mathrm{K}(<\mathrm{M})$ eigenimages are selected to correspond to the $\mathrm{K}$ highest eigenvalues. The set of images, $\{\mathrm{i}\}$ is transformed into its eigenimage components (projected into the image space) by the operation: $\omega_{\mathrm{nk}}=\mathrm{b}_{\mathrm{k}}\left(i_{n}-\hat{i}\right)$ where $\mathrm{n}=1, \ldots, \mathrm{M}$ and $\mathrm{k}=1, \ldots, \mathrm{K}$. The weights $\Omega \mathrm{n}=$ $\omega_{n 1}, \omega_{n 2, \ldots \ldots . . . \omega_{n k}}$ that describes the contribution of each eigenimage in representing the input palm /face image, treating eigen palms/face as a basis set for images.

\section{FEATURE LEVEL FUSION}

Let $F_{i}=\left\{f_{i, 1}, f_{i, 2}, \ldots f_{i, n}\right\}$ and $P_{i}=\left\{p_{i, 1}, p_{i, 2}, \ldots p_{i, m}\right\}$ represent the feature vector of the face (Eigen coefficients ) and palm modalities of a user, respectively. The fused feature vector $X i$ $=\left\{x_{i}, 1, x_{i}, 2 \ldots x_{i}, d\right\}$ can be obtained by augmenting the normalized feature vectors $F_{i^{\prime}}$ and $P_{i^{\prime}}$ and performing feature selection on the concatenated vector. The simple sequential forward search can be used for this.

\section{A. Need for Normalization}

The individual feature values of vectors $F_{i}=\left\{f_{i, 1}, f_{i, 2}, \ldots f_{i, n}\right.$ \} and $P_{i}=\left\{p_{i, 1}, p_{i, 2}, \ldots p_{i, m}\right\}$ may exhibit significant differences in their range as well as form (i.e., distribution). Concatenating such diverse feature values will not be appropriate in many cases. For example, if the $F_{i}$ 's are in the range [0], [100] while the $\mathrm{P}_{\mathrm{i}}$ 's are in the range [0], [1], then the distance between two concatenated feature vectors will be more sensitive to the $F_{i}$ 's than the $P_{i}$ 's. The goal of feature normalization is to modify the location (mean) and scale (variance) of the features values via a transformation function in order to map them into a common domain. The min-max normalization scheme can be used for normalization.

\section{B. Min Max Normalization Scheme}

Let $\mathrm{p}$ and $\mathrm{p}^{\prime}$ denote a feature value before and after normalization, respectively. The min-max technique computes $\mathrm{p}$ as ' $p^{\prime}=\frac{p-\min \left(f_{p}\right)}{\max \left(f_{p}\right)-\min \left(f_{p}\right)} \quad$ where $f_{p}$ is the function which generates $\mathrm{p}$, and $\min \left(f_{p}\right)$ and $\max \left(f_{p}\right)$ represent the minimum and maximum of all possible $p$ values that will be observed, respectively. Estimate of these parameters has to be obtained from the available set of training data. Feature normalization may not be necessary in cases where the feature values pertaining to multiple sources are already comparable.

\section{Fusion Algorithm}

Consider feature vectors $\left\{F_{i}, P_{i}\right\}$ and $\left\{F_{j}, P_{j}\right\}$ obtained at two different time instances $i$ and $\mathrm{j}$. The corresponding fused feature vectors may be denoted as $X_{i}$ and $X j$, respectively. Let $\mathrm{SF}$ and SP be the normalized match (distance) scores [7] generated by comparing $F i$ with $F j$ and $P i$ with $P_{j}$, respectively and let $S_{f u s}=(S F+S P) / 2$ be the fused match score obtained using the simple sum rule. The algorithm first determines if $S_{f u s}$ falls in the critical region, C, of the match score distribution. The critical region is defined as a range of scores, $[t-\varepsilon, t+\varepsilon]$, where the probability distributions of the genuine and impostor scores have substantial overlap. Match scores which occur below (above) C can be (almost) definitively stated to be genuine (impostor) scores. However, $S_{f u s}$ within C presents an ambiguous situation. Thus, the fused vectors $X_{i}$ and $X_{j}$ are also used in the decision process. In the case of genuine pairs, a high match score is typically the effect of a few feature values constituting the vector while a similar score for an impostor pair is typically the cumulative effect of all feature values. Thus, two distance measures are considered to distinguish genuine and impostor pairs, the Euclidean distance, $S_{e u c}=\sum_{k=1}^{d}\left(x_{i, k}-x_{j, k}\right)^{2}$ and the feature distance, $S_{\text {feat }}=\sum_{k=1}^{d} I\left(\left|x_{i, k}-x_{j, k}\right|\right)$ where I (.) is the indicator function such that $\mathrm{I}(\mathrm{y})=1$, if $\mathrm{y}>\mathrm{t} 1$ (and 0 otherwise). Now $S_{\text {feat }}$ and $S_{\text {euc }}$ along with $S_{f u s}$ are used to arrive at the final decision. This technique is termed as the feedback technique since a feedback routine is implemented between the feature extraction and the matching modules.

\section{IDENTIFICATION}

When the probe images i.e., face and palmprint of a person is captured, its features are extracted, normalized and fused in above manner. The various distances like $S_{f u s}, S_{\text {euc }}$, and ${ }_{\text {Sfeat }}$ are calculated and compared with stored distances in database as explained above. The person is identified with his stored name and address in the database.

\section{CONCLUSION}

Biometric systems are widely used to overcome the traditional methods of authentication. But the unimodal biometric system fails in case of biometric data for particular trait. Combining the two traits definitely enhances security. This paper has presented a feature level fusion system of face and palmprint traits using a simple fusion algorithm. Since feature set contains relevant and richer information about the captured biometric evidence, fusion at feature level is expected to provide more accurate results as compared to other fusion methods. The GAR using palm images only is found to be $81.48 \%$. The GAR using face images is found to be $88.88 \%$. The fusion results indicate substantial increase in GAR as compared to GAR obtained using only palm and only face modality and gives overall accuracy more than $95 \%$.

\section{REFERENCES}

[1] A. Ross, A.K. Jain, and J. Z. Qian, "Information Fusion in Biometrics," in 3rd International Conference on Audio- and Video-Based Person Authentication (AVBPA), Sweden, pp. 354 - 359. 2001.

[2] D. R. Kisku, P. Gupta, and J. K. Sing, "Feature Level Fusion of Biometric Cues: Human Identification with Doddington's Caricature,' in International Conference on Security Technology, Communications in Computer and Information Sciences, pp. 157 - 164, SpringerVerlag 2009 
[3] A. Rattani, D. R. Kisku, M. Bicego, and M. Tistarelli, "Feature Level Fusion of Face and Fingerprint Biometrics," 1st IEEE International Conference on Biometrics, Theory, Applications and Systems, pp. $1-6$ September 2007.

[4] Handbook of Multibiometrics, A. Ross, K. Nandakumar, and A. K. Jain, Springer Verlag 2006, pp. 59-91.

[5] A. K. Jain, A. Ross, and S. Prabhakar, "An Introduction to Biometric Recognition," IEEE Transactions on Circuits and Systems for Video Technology, Special Issue on Image- and Video-Based Biometrics, vol. 14(1), pp. 4-20, January 2004.

[6] M. Nageshkumar, P. K Mahesh, and M. N. Shanmukha Swamy. (August 2009). An Efficient Secure Multimodal Biometric Fusion Using Palmprint and Face Image. IJCSI International Journal of Computer Science Issues, Vol. 2, pp. 49-53. Available: http://www.ijcsi.org/papers/VOLUME_2.pdf, ISSN (Online): 1694-0784

[7] A. Ross and R. Govindarajan, "Feature Level Fusion in Biometric Systems," SPICE Conference on Biometric Technology for human identificatio. II ,Vol. 5779, Orlando USA, March 2005, pp,.196-204.

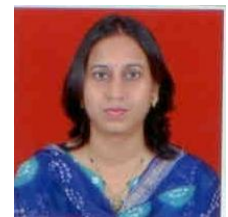

Gayatri Umakant Bokade, graduated in Electronics and Telecommunication from Amravati University in 1997 and received M.Tech degree in Electronics and Telecommunication with specialization Wired and Wireless Communication from College of Engineering Pune, during 2009-2011.

She is currently working as an assistant professor in ENTC Dept at Nutan Maharashtra Institute of Engineering and Technology, Talgaon dabhade, Dist. Pune and presented two papers in National Conference and two papers in International Conference. All the papers are based on biometric user authentication.

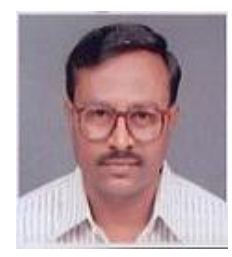

Ashok M. Sapkal, graduated in Electronics and Telecommunication from University of Pune in 1987, received M.E degree in Electronics and Telecommunication from College of Engineering Pune, University of Pune in 1992 and completed PhD in the same field in 2008 from university of Pune.

$\mathrm{He}$ is currently working as professor in ENTC Dept at College of Engineering Pune and presented eight papers in National Conference, two in National journal, twenty in International Conference, eleven in International journals and five papers are available on IEEE Explorer. 